\title{
Microscopic positive-energy potential based on Gogny interaction
}

\author{
G. Blanchon ${ }^{(1)}$, M. Dupuis ${ }^{(1)}$, H. F. Arellano ${ }^{(1,2)}$, and N. Vinh Mau \\ (1) CEA,DAM,DIF F-91297 Arpajon, France and \\ (2) Department of Physics - FCFM, University of Chile, Av. Blanco Encalada 2008, Santiago, Chile
}

\begin{abstract}
We present nucleon elastic scattering calculation based on Green's function formalism in the Random-Phase Approximation. For the first time, the Gogny effective interaction is used consistently throughout the whole calculation to account for the complex, non-local and energy-dependent optical potential. Effects of intermediate single-particle resonances are included and found to play a crucial role in the account for measured reaction cross section. Double counting of the particlehole second-order contribution is carefully addressed. The resulting integro-differential Schrödinger equation for the scattering process is solved without localization procedures. The method is applied to neutron and proton elastic scattering from ${ }^{40} \mathrm{Ca}$. A successful account for differential and integral cross sections, including analyzing powers, is obtained for incident energies up to $30 \mathrm{MeV}$. Discrepancies at higher energies are related to much too high volume integral of the real potential for large partial waves. Moreover, this works opens the way for future effective interactions suitable simultaneously for both nuclear structure and reaction.
\end{abstract}

Nuclear structure and nuclear reactions are two aspects of the same many-body problem, although in practice they are often addressed as different phenomena. A consistent, quantitative and predictive account for both is still a challenging open problem in nuclear physics. The description of nucleon-nucleus elastic scattering based solely on the nucleon-nucleon (NN) interaction is an important step forward toward this unification.

Depending on projectile energy and target mass, various strategies have been adopted in order to treat microscopically elastic scattering. Nuclear matter models [1] provide reasonable descriptions of nucleon elastic scattering at incident energies above $50 \mathrm{MeV}$ [2], even up to $\sim 1 \mathrm{GeV}$ [3]. The Resonating Group Method within the No-Core Shell Model, has successfully described nucleon and deuteron scattering from light nuclei [4]. These models have recently been extended to include three-nucleon forces for nucleon scattering from ${ }^{4} \mathrm{He}$ [5]. The Green's Function Monte Carlo method has been used to describe elastic scattering from ${ }^{4} \mathrm{He}[6]$. These models yield encouraging results but are still restricted to light targets at low energies. The Self-Consistent Green's Function (SCGF) method has been applied to microscopic calculation of the optical potentials for proton scattering from ${ }^{16} \mathrm{O}[7,8]$. The coupled-cluster theory has been applied to proton elastic scattering from ${ }^{40} \mathrm{Ca}$ [9]. These last two methods are limited to closed-shell nuclei. Work on Gorkov-Green's function theory is in progress to extend SCGF to nuclei around closed-shell nuclei [10, 11]. An alternative method consists of using microscopic approaches based on the self-consistent mean-field theory and its extensions beyond mean-field. In nuclear physics, they are usually based on energy density functionals built from phenomenological parametrizations of the NN effective interaction, such as Skyrme [12, 13] or Gogny forces [14 17]. These approaches have successfully predicted a broad body of nuclear structure observables for nuclei ranging from medium to heavy masses. This wealth of developments can be extended to reaction calculations based on NN effective interaction. The so-called Nuclear Structure Method (NSM) for scattering [18 22] relies on the self-consistent Hartree-Fock (HF) and RandomPhase Approximations (RPA) of the microscopic optical potential. The former is a mean-field potential and the latter is a polarization potential built from targetnucleus excitations. A strictly equivalent method, the continuum particle-vibration coupling using a Skyrme interaction, has been recently applied to neutron scattering from ${ }^{16} \mathrm{O}$ [23], but neglecting part of the residual interaction in the coupling vertices. Other approaches are in progress, where optical potential is approximated as the HF term plus the imaginary part of the uncorrelated particle-hole potential neglecting the collectivity of target excited states [24, 25].

We report on optical potential calculations using NSM 18]. Here the optical potential $V$ consists of two components,

$$
V=V^{H F}+\Delta V
$$

The HF potential, $V^{H F}$, is the major contribution to the real part of the optical potential. $V^{H F}$ is calculated in coordinate space to ensure the correct asymptotic behavior of single-particle states. It is non local and energy independent due to the nature of Gogny interaction, which is of finite range and energy independent, respectively. Rearrangement contributions stemming from the density-dependent term of the interaction are also taken into account.

The second component of the potential in Eq. (1) is

$$
\Delta V=V^{P P}+V^{R P A}-2 V^{(2)},
$$

which is complex, energy dependent and non local. Here $V^{P P}$ and $V^{R P A}$ are contributions from particle-particle and particle-hole correlations, respectively. The uncorrelated particle-hole contribution $V^{(2)}$ is accounted for 
once in $V^{P P}$, and twice in $V^{R P A}$. As a matter of fact, if two-body correlations are neglected in Eq. (2) for $V^{P P}$ and $V^{R P A}$, then $\Delta V$ reduces to $V^{(2)}$ as expected [18].

As mentioned in Ref. 21], if one works with an NN effective interaction with a density-dependent term, such as Gogny or Skyrme forces, attention must be paid to correlations already accounted for in the interaction. Indeed, part of particle-particle correlations is already contained at the HF level as far as $\operatorname{Re}\left[V^{P P}\right]$ is concerned. We thus use the same prescription as in Ref. 20], omitting $\operatorname{Re}\left[V^{P P}\right]$ while $\operatorname{Im}\left[V^{P P}\right]$ is approximated by $\operatorname{Im}\left[V^{(2)}\right]$. Then Eq. (2) becomes

$$
\Delta V=\operatorname{Im}\left[V^{(2)}\right]+V^{R P A}-2 V^{(2)} .
$$

From now on, equations are presented omitting spin for simplicity. For nucleons with incident energy E, the RPA potential reads

$$
\begin{aligned}
V^{R P A}\left(\mathbf{r}, \mathbf{r}^{\prime}, E\right) & =\sum_{N \neq 0} y_{\lambda}\left[\frac{n_{\lambda}}{E-\varepsilon_{\lambda}+E_{N}-i \Gamma\left(E_{N}\right)}\right. \\
& \left.+\frac{1-n_{\lambda}}{E-\varepsilon_{\lambda}-E_{N}+i \Gamma\left(E_{N}\right)}\right] \\
& \times \Omega_{\lambda}^{N}(\mathbf{r}) \Omega_{\lambda}^{N}\left(\mathbf{r}^{\prime}\right),
\end{aligned}
$$

where $n_{i}$ and $\varepsilon_{i}$ are occupation number and energy of the single-particle state $\phi_{i}$ in the HF field, respectively. Subscripts $p, h$ and $\lambda$ refer to the quantum number of particle, hole and the intermediate single-particle, respectively. $E_{N}$ and $\Gamma\left(E_{N}\right)$ represent the energy and the width of the $N^{t h}$ excited state of the target, respectively. Additionally

$$
\Omega_{\lambda}^{N}(\mathbf{r})=\sum_{(p, h)}\left[X^{N,(p, h)} F_{p h \lambda}(\mathbf{r})+Y^{N,(p, h)} F_{h p \lambda}(\mathbf{r})\right],
$$

where $X$ and $Y$ denote the usual RPA amplitudes and

$$
F_{i j \lambda}(\mathbf{r})=\int d^{3} \mathbf{r}_{1} \phi_{i}^{*}\left(\mathbf{r}_{1}\right) v\left(\mathbf{r}, \mathbf{r}_{1}\right)[1-\hat{\mathrm{P}}] \phi_{\lambda}(\mathbf{r}) \phi_{j}\left(\mathbf{r}_{1}\right),
$$

where $\hat{\mathrm{P}}$ is a particle-exchange operator and $v$ is the NN effective interaction. The particle-hole contribution reads

$$
\begin{aligned}
V^{(2)}\left(\mathbf{r}, \mathbf{r}^{\prime}, E\right) & =\frac{1}{2} \sum_{i j} \sum_{\lambda}\left[\frac{n_{i}\left(1-n_{j}\right) n_{\lambda}}{E-\varepsilon_{\lambda}+E_{i j}-i \Gamma\left(E_{i j}\right)}\right. \\
& \left.+\frac{n_{j}\left(1-n_{i}\right)\left(1-n_{\lambda}\right)}{E-\varepsilon_{\lambda}-E_{i j}+i \Gamma\left(E_{i j}\right)}\right] \\
& \times F_{i j \lambda}(\mathbf{r}) F_{i j \lambda}^{*}\left(\mathbf{r}^{\prime}\right),
\end{aligned}
$$

with $E_{i j}=\varepsilon_{i}-\varepsilon_{j}$, the uncorrelated particle-hole energy.

The description of target excitations has been obtained by solving the RPA/D1S equations in a harmonic oscillator basis, including fifteen major shells [26] and using the D1S Gogny interaction [15]. We account for RPA excited states with spin up to $J=8$, including both parities in order to achieve convergence of the cross section. The first $1^{-}$state given by RPA, containing the translational spurious mode, is removed. In order to avoid spurious modes in the uncorrelated particle-hole term, we approximate the $1^{-}$contribution in $V^{(2)}$ by half that of the $1^{-}$contribution in $V^{R P A}$. Coupling to excited states results in a number of poles in Eqs. (4) and (77). Moreover, fluctuations appear in the imaginary part of the potential whenever the energy $E-E_{N}$ matches a resonance energy of the intermediate single-particle state $\phi_{\lambda}$. The leading inelastic doorways are those containing singleparticle resonances. Although the RPA/D1S method provides a good overall description of the spectroscopic properties of double-closed shell nuclei, couplings to two or more particle-hole states and to continuum states are neglected. The impact of these couplings is a strength redistribution that can be handled assigning a finite width $\Gamma\left(E_{N}\right)$ to each RPA state. It has the effect of averaging in energy and smoothing the potential. The resulting potential can then be identified with an optical model [27]. A microscopic calculation of these widths is beyond the scope of the present study. We include them phenomenologically as an interpolation between reasonable values. $\Gamma\left(E_{N}\right)$ takes the value of $2,5,15$ and $50 \mathrm{MeV}$, for excitation energies of 20, 50, 100 and $200 \mathrm{MeV}$, respectively. The integro-differential Schrödinger equation for elastic scattering is solved retaining the non-local structure of the potential [28]. Moreover optical potential calculations yield shape elastic, reaction and total cross sections [27]. The compound-elastic cross section has to be added to shape elastic cross section and subtracted from reaction cross section before comparison with data 27. In a first attempt, we use the compound-elastic contribution from Hauser-Feshbach calculations with TALYS code 29] using Koning-Delaroche global potential [30]. These considerations are particularly relevant for neutron scattering below $10 \mathrm{MeV}$.

In Fig. 1] we present results for the calculated differential cross sections based on NSM for both neutron and proton scattering from ${ }^{40} \mathrm{Ca}$. References to data are given in Ref. [30]. Error bars are smaller than the size symbols. NSM results compare very well to experiment and those based on Koning-Delaroche potential up to about $30 \mathrm{MeV}$ incident energy. Beyond $30 \mathrm{MeV}$, backwardangle cross sections are overestimated. Discrepancies at 16.9 MeV (23.5 MeV) for neutron (proton) scattering are related to resonances in the intermediate single-particle state when not completely averaged. A detailed treatment of the width might cure this issue. In Fig. 2 we show calculated analyzing powers for neutron and proton scattering at several energies, in good agreement with measurements. Moreover, agreement with the data is comparable to that obtained from Koning-Delaroche potential. These results suggest that NSM potential retains the correct spin-orbit behavior. In Fig. 3 we show reac- 

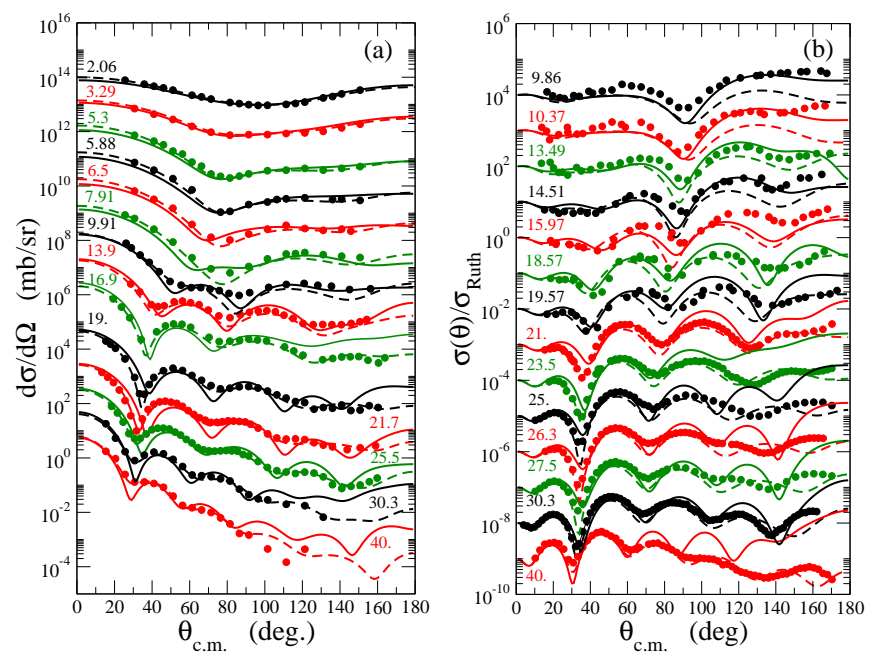

FIG. 1: Differential cross sections for neutron (a) and proton (b) scattering from ${ }^{40} \mathrm{Ca}$. Comparison between data (symbols), $V^{H F}+\Delta V$ results (solid curves) and Koning-Delaroche potential results (dashed curves).
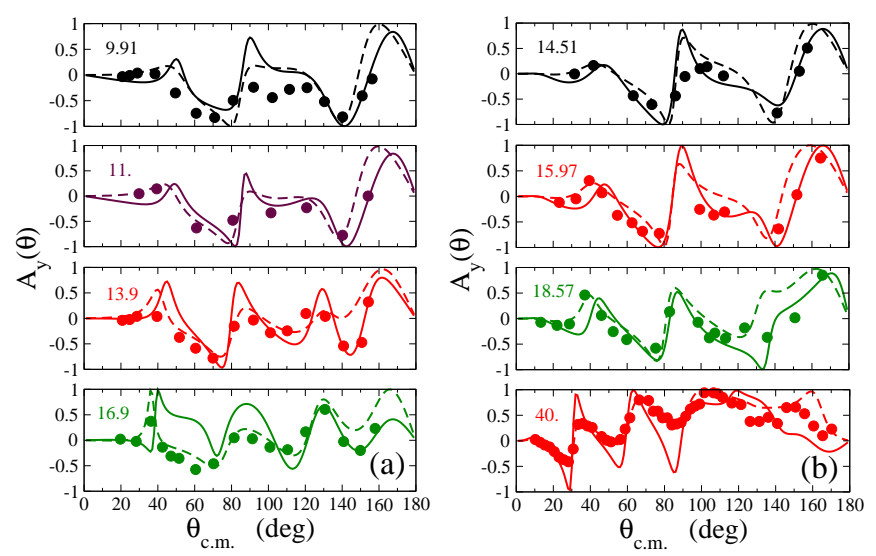

FIG. 2: Same as Fig. 1 for analyzing powers.

tion cross section for proton scattering (a) and total cross section for neutron scattering (b). Calculated reaction cross sections are in good agreement with experiments. For neutrons, however, we underestimate the total cross section below $10 \mathrm{MeV}$. Considering that the differential elastic cross section is well reproduced, this underestimate suggests that part of the absorption mechanism is not accounted for, as target-excited states beyond RPA or double-charge exchange process.

To understand the limited energy range of applicability of the NSM approach, we compare in Fig. प the volume integral, $J_{V}$, of the central HF potential with the one obtained from the real part of the Perey-Buck non-local potential 31]. Black segments denote the strongest partialwave contributions accounting for $80 \%$ of the reaction cross section at the selected incident energies. Keep in mind that the HF potential is the leading contribution to the real part of $V$ in Eq. (1). Its contribution to
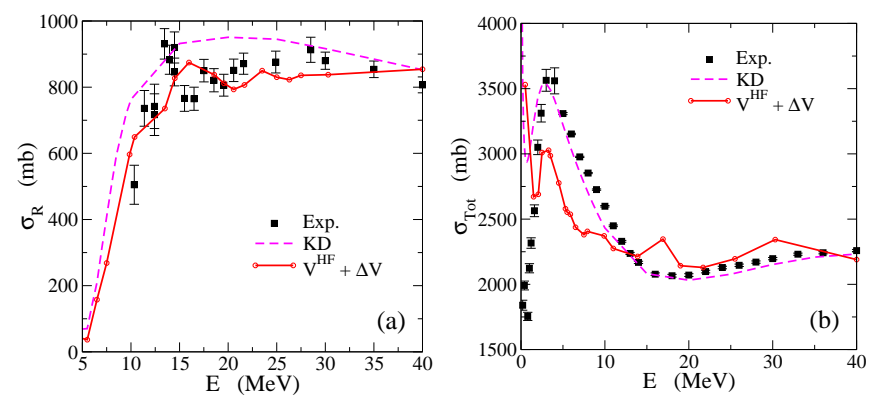

FIG. 3: Reaction cross section for proton (a) and total cross section for neutron (b) scattering from ${ }^{40} \mathrm{Ca}$. Comparison between data (symbols), $V^{H F}+\Delta V$ results (solid curve) and Koning-Delaroche potential (dashed curve).

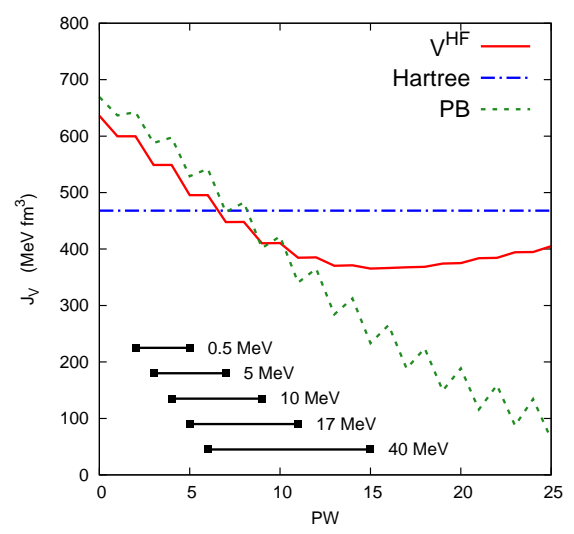

FIG. 4: Volume integral as a function of partial waves for neutron scattering from ${ }^{40} \mathrm{Ca}$ : HF potential (solid curve), Hartree potential (dash-dotted curve) and Perey-Buck potential (dotted curve). Horizontal segments denote the partial-wave interval to sum up $80 \%$ of the reaction cross section at selected incident energies.

$J_{V}$ is similar to that from Perey-Buck up to about the twelfth partial wave $(\sim 17 \mathrm{MeV})$. Beyond this point $\mathrm{HF}$ saturates, following the trend of the Hartree potential which is local and thus partial-wave independent. This departure from Perey-Buck explains why increasing incident energy (partial wave) yields much too high $J_{V}$ for $\mathrm{HF}$, with the subsequent overestimate of the differential cross section at backward angles. It would be interesting to investigate to what extent the effective interaction has incidence on this behavior at high partial wave.

We now address the subtraction of the uncorrelated second-order term in Eq. (22). As pointed out in Ref. [32], this subtraction can lead to unphysical solutions with spurious poles and negative occupation numbers. The smooth and averaged potential obtained from Eq. (1) no longer suffers these pathologies. Indeed if one approximates $V^{(2)} \approx V^{R P A} / 2$, then Eq. (2) reduces to

$$
\Delta V \approx \operatorname{Im}\left[V^{R P A} / 2\right] \text {. }
$$

This approximation has the drawback of neglecting the real part of $\Delta V$ as well as part of the collectivity of the 
excited states. However, it has the advantage of avoiding second-order double counting. As seen in Fig. 5 both

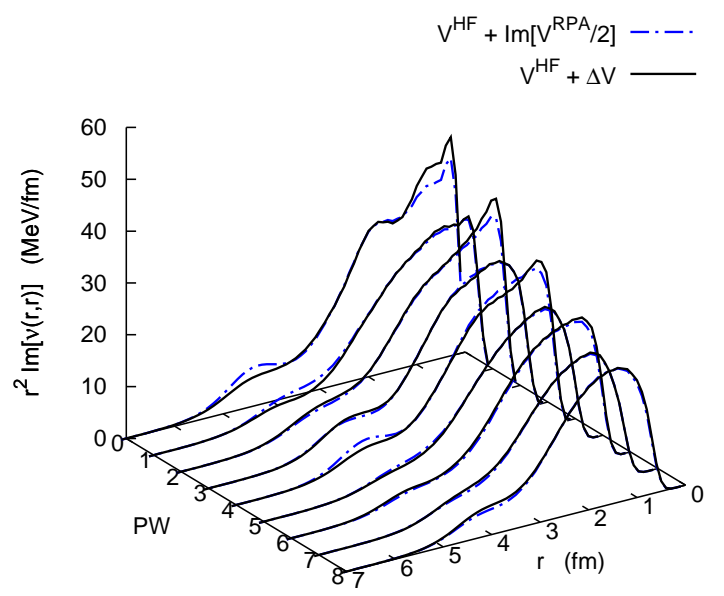

FIG. 5: Partial-wave contribution $\nu$ of $\operatorname{Im} V$ for neutron scattering from ${ }^{40} \mathrm{Ca}$ scattering at $9.91 \mathrm{MeV}$ as a function of radius and partial waves: $V^{H F}+\Delta V$ potential (solid curve), $V^{H F}+\operatorname{Im}\left[V^{R P A} / 2\right]$ potential (dash-dotted curve).

approximations in Eqs. (3) and (8) yield very similar shapes for each partial-wave contribution $\nu$ of the diagonal imaginary part of $\operatorname{Im}[V]$ for neutron scattering from ${ }^{40} \mathrm{Ca}$ at $9.91 \mathrm{MeV}$. This trend remains true for higher partial waves and incident energies, confirming the good behavior of $V^{H F}+\Delta V$. In Fig. [6] we present the differential cross section for proton scattering from ${ }^{40} \mathrm{Ca}$ based on these two approximations. The diffractive minima obtained with $V^{H F}+\Delta V$ agree better with experiment than those obtained from $V^{H F}+\operatorname{Im}\left[V^{R P A} / 2\right]$. This result emphasizes the important role played by the real part of $\Delta V$.

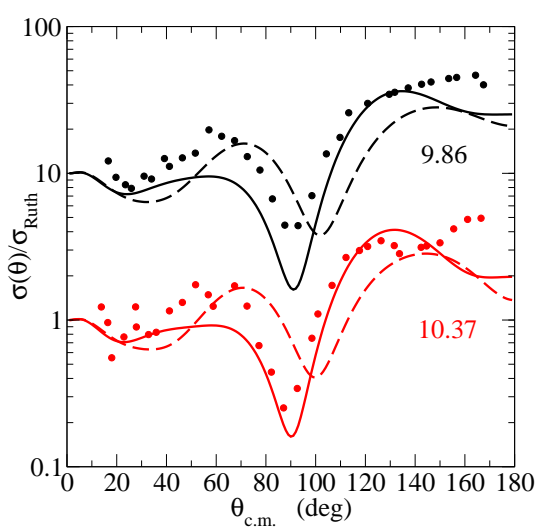

FIG. 6: Differential cross sections $\sigma(\theta) / \sigma_{\text {Ruth }}$ for proton incident on ${ }^{40} \mathrm{Ca}$. Comparison between data (symbols), $V^{H F}+\Delta V$ (solid curves) and $V^{H F}+\operatorname{Im}\left[V^{R P A} / 2\right]$ results (dashed curves).

The work presented here constitutes a promising step forward aimed at a model keeping at the same footing both reaction and structure aspects of the many-nucleon system. Within the optical model potential, NSM is able to account reasonably well for low energy scattering data. An important feature of the approach is the extraction of the imaginary part of the potential by means of intermediate excitations of the system. It has been based on Gogny effective interaction, although it can be applied to any interaction of similar nature. The study has been restricted to closed-shell targets but can be extended to deformed nuclei described with Quasi-particle RPA. Those results also open the way to new parametrizations of $\mathrm{NN}$ effective interactions including reaction phenomena. A comprehensive work on the formalism and applications shall be presented elsewhere.

H. F. A. acknowledges partial funding from FONDECYT under Grant No 1120396.

[1] J. Hufner and C. Mahaux, Ann. Phys. (NY) 73, 525 (1972).

[2] M. Dupuis, S. Karataglidis, E. Bauge, J. P. Delaroche, and D. Gogny, Phys. Rev. C 73, 014605 (2006).

[3] H. F. Arellano and H. V. von Geramb, Phys. Rev. C 66, 024602 (2002).

[4] S. Quaglioni and P. Navrátil, Phys. Rev. Lett. 101, 092501 (2008).

[5] G. Hupin, J. Langhammer, P. Navrátil, S. Quaglioni, A. Calci, and R. Roth, Phys. Rev. C 88, 054622 (2013).

[6] K. M. Nollett, S. C. Pieper, R. B. Wiringa, J. Carlson, and G. M. Hale, Phys. Rev. Lett. 99, 022502 (2007).

[7] H. Dussan, S. J. Waldecker, W. H. Dickhoff, H. Müther, and A. Polls, Phys. Rev. C 84, 044319 (2011).

[8] C. Barbieri and B. K. Jennings, Phys. Rev. C 72, 014613 (2005).

[9] G. Hagen and N. Michel, Phys. Rev. C 86, 021602 (2012).

[10] V. Somà, C. Barbieri, and T. Duguet, Phys. Rev. C 87, 011303 (2013).

[11] V. Somà, C. Barbieri, and T. Duguet, Phys. Rev. C 89, 024323 (2014).

[12] D. Vautherin and D. M. Brink, Phys. Rev. C 5, 626 (1972).

[13] V. Hellemans, A. Pastore, T. Duguet, K. Bennaceur, D. Davesne, J. Meyer, M. Bender, and P.-H. Heenen, Phys. Rev. C 88, 064323 (2013).

[14] J. Dechargé and D. Gogny, Phys. Rev. C 21, 1568 (1980).

[15] J. F. Berger, M. Girod, and D. Gogny, Comput. Phys. Commun. 63, 365 (1991).

[16] F. Chappert, M. Girod, and S. Hilaire, Phys. Lett. B 668, 420 (2008).

[17] S. Goriely, S. Hilaire, M. Girod, and S. Péru, Phys. Rev. Lett. 102, 242501 (2009).

[18] N. Vinh Mau, Theory of nuclear structure (IAEA, Vienna) p. 931 (1970).

[19] N. Vinh Mau and A. Bouyssy, Nucl. Phys. A257, 189 (1976), ISSN 0375-9474.

[20] V. Bernard and N. Van Giai, Nucl. Phys. A327, 397 (1979).

[21] A. Bouyssy, H. Ngô, and N. Vinh Mau, Nucl. Phys. A371, 173 (1981). 
[22] F. Osterfeld, J. Wambach, and V. A. Madsen, Phys. Rev. C 23, 179 (1981).

[23] K. Mizuyama and K. Ogata, Phys. Rev. C 86, 041603 (2012).

[24] V. V. Pilipenko and V. I. Kuprikov, Phys. Rev. C 86, 064613 (2012).

[25] Y. Xu, H. Guo, Y. Han, and Q. Shen, J. Phys. G: Nucl. Part. Phys. 41, 015101 (2014).

[26] J. Blaizot and D. Gogny, Nucl. Phys. A284, 429 (1977).

[27] H. Feshbach, Annals of Physics 5, 357 (1958).

[28] J. Raynal, computer code DWBA98, 1998, (NEA
$1209 / 05)$.

[29] A. J. Koning, S. Hilaire, and M. Duijvestijn, in Proceeding of the International Conference on Nuclear Data for Science and Technology-ND2007 (EDP $\quad$ Sciences, Paris, France, 2008), pp. 211-214.

[30] A. J. Koning and J. P. Delaroche, Nucl. Phys. A713, 231 (2003).

[31] F. Perey and B. Buck, Nucl. Phys. 32, 353 (1962).

[32] C. Barbieri and W. H. Dickhoff, Phys. Rev. C 63, 034313 (2001). 